\title{
La televisión de los estados provinciales: oferta cultural mainstream o diversidad cultural
}

\author{
The States provincial TV channels: offer cultural mainstream or \\ cultural diversity
}

Hernán Pajoni hpajoni@gmail.com

http://orcid.org/0000-0001-8365-1121

Facultad de Ciencias Sociales; Universidad de Buenos Aires/

Universidad Católica Argentina (Argentina)

\section{Resumen}

El trabajo analiza la oferta de contenidos en la programación de los canales abiertos de televisión gestionados por los estados provinciales para compararlos en cada caso con la programación del canal privado en la misma área de cobertura. La particular forma de constitución del sistema televisivo en la Argentina da resultados singulares a raíz de la ausencia de principios de carácter educativo y cultural en su génesis, que constituyen los ideales de las formas estatales en la gestión de medios de comunicación. Los acuerdos de programación entre las emisoras estatales y los canales privados de televisión abierta de la Ciudad de Buenos Aires son una característica fundamental del modelo de radiodifusión.

Palabras clave: Televisión; radiodifusión; programación; cultura; Estado. 


\begin{abstract}
The work analyzes the content offer in the programming of the channels of television managed by the provincial states to compare them in every case with the programming of the private channels in the same area of coverage. The particular form of constitution of the television system in Argentina gives singular results because of the absence of educational and cultural values in the management of mass media. The agreements of programming between state channels and the private television of the City of Buenos Aires are a fundamental aspect of the broadcasting model.
\end{abstract}

Keywords: Television; broadcasting; programming; culture; State.

En la historia de la radiodifusión en la Argentina, la relación entre el Estado y los prestadores privados titulares de licencia tiene una complejidad que excede una lectura sencilla acerca de la interacción entre regulador y regulado. Esta relación adquiere una dinámica muy particular cuando se analiza la intervención de los propios estados provinciales como prestadores de servicios de radiodifusión.

Los estados locales y nacionales que operan licencias de radiodifusión están sometidos a un conjunto de exigencias de origen político-académico vinculadas al cumplimiento de objetivos culturales no mercantiles, en torno al ideal del modelo europeo de servicio público que inspiró principios democráticos, de calidad, independencia y representación multicultural. Las políticas de radiodifusión en la Argentina vistas desde la perspectiva del rol del Estado como prestador de servicios de radiodifusión pone en evidencia, con una simple lectura, las dificultades para constituirse como actores relevantes en la producción y distribución de contenidos en el marco de un sistema privado, concentrado y altamente centralizado.

Tal complejidad implica abordar una historización de nuestras contradicciones y conflictos en la conformación del Estado nacional y la formación de los poderes económicos locales que detentaron el sistema de radiodifusión desde sus inicios. Este derrotero de análisis -desde las postrimerías de las primeras emisiones radiales a partir de 1920- está bien documentado por numerosas investigaciones y publicaciones académicas que despejan, desde diferentes perspectivas y términos teóricos- las complejidades de esa historia y por tanto de nuestro presente. 
Este trabajo se propone relevar la propuesta de contenidos de la programación de cada canal abierto gestionado por los estados provinciales para compararlo con la programación de los prestadores privados en cada jurisdicción. Esta indagación permitirá establecer diferencias y/o semejanzas entre las propuestas culturales del Estado local frente al licenciatario privado con fines de lucro, y concluir acerca de la oferta cultural de los estados provinciales frente a los privados.

\section{Sobre la creación de los canales de los estados provinciales}

La creación de canales abiertos de televisión controlados por los estados provinciales obedece a muy distintas razones: económicas, geográficas, políticas, de infraestructura técnica y en muchos casos relacionados con una necesidad concreta de incrementar el acceso de la población a la oferta cultural. De este modo, "tanto los modelos de gestión como las decisiones de programación son tan heterogéneas como cada una de las motivaciones que marcaron el inicio de las transmisiones, su consolidación y evolución". (Pajoni, 2017).

Hasta 1980 en el país existían once canales de televisión estatales, además de Canal 7 y sus repetidoras: Canal 10 de Córdoba, Canal 10 de Tucumán, Canal 13 de Río Grande (Tierra del Fuego), Canal 11 de Ushuaia (Tierra del Fuego), Canal 3 de la provincia de La Pampa, Canal 9 de Río Gallegos (Santa Cruz), Canal 12 de Trenque Lauquen (Municipalidad de Trenque Lauquen, provincia de Buenos Aires), Canal 9 de La Rioja, Canal 13 de San Luis, Canal 12 de Posadas (Misiones) y Canal 11 de Formosa.

Luego se sumaron en Canal 10 de Río Negro en 1985; Canal 7 de Rawson (Chubut) en 1990, Canal 7 de Tartagal (Salta) en 1992. Recién 17 años después, y en el marco de la ley de Servicios de Comunicación Audiovisual aprobada en 2009, comenzó a emitir en 2010 Chaco TV; en 2013 Acequia TV de la provincia de Mendoza y un año más se creó Catamarca Radio y Televisión Sociedad del Estado. Por su parte, una iniciativa aún demorada es la de Radio y televisión santafecina que inició sus transmisiones en 2016 sólo por internet pese a las promesas de transformarse en el primer canal provincial nacido a la luz del modelo de gestión de la radiodifusión pública establecido por Ley de Servicios de Comunicación Audiovisual 26522/2009.

En particular, y con respecto a la conformación del sistema de radiodifusión en cada provincia, es indispensable hacer referencia a la centralización política de las decisiones en materia de otorgamiento de licencias en todo el territorio argentino, que siempre estuvo bajo la atribución 
exclusiva de la autoridad de aplicación federal en la responsabilidad de las asignaciones de frecuencia, sobre todas las jurisdicciones o áreas de cobertura del país. Esta condición trae aparejada la dificultad estructural de la implementación de un diseño autónomo de políticas provinciales en materia de radiodifusión y producción cultural.

\begin{tabular}{|l|l|}
\hline \multicolumn{2}{|c|}{17 canales de televisión abierta de los estados provinciales } \\
\hline Canal & Año de creación \\
\hline Canal 10 de Córdoba & 1962 \\
\hline Canal 10 de Tucumán & 1966 \\
\hline Canal 13-Río Grande-Ushuaia & 1967 \\
\hline Canal 11-Ushuaia Tierra del Fuego & 1967 \\
\hline Canal 9 Río Gallegos-Santa Cruz & 1968 \\
\hline Canal 12 de Trenque Lauquen-1737 & 1970 \\
\hline Canal 9 de La Rioja & 1970 \\
\hline Canal 12 de Posadas, Misiones & 1972 \\
\hline Canal 3 de la provincia de La Pampa & 1972 \\
\hline Canal 13 de la provincia de San Luis & 1972 \\
\hline Canal 7 Rawson, provincia de Chubut & 1975 \\
\hline Lapacho TV, Canal 11 de Formosa & 1978 \\
\hline Canal 10-Televisión rionegrina & 1985 \\
\hline Canal 3 Formosa Televisión Color (FTC) de la provincia de Formosa & 1987 \\
\hline Chaco TV. Resistencia, Chaco & 2010 \\
\hline Canal Acequia TV, Mendoza & 2013 \\
\hline Catamarca Radio y Televisión Sociedad del Estado & 2014 \\
\hline Radio y televisión santafecina no se contabiliza como canal abierto & emite por internet \\
\hline
\end{tabular}

Desde su creación, los canales abiertos de la Patagonia argentina, Canal 13 de Río Grande y Canal 11 de Ushuaia -este último hasta 2013- reprodujeron la programación de Canal 11 y Canal 13 de la Ciudad de Buenos Aires respectivamente. En el caso de Canal 11 de Ushuaia, a partir de 2013, produjo contenidos propios y principalmente con los que les proveía el gobierno nacional a través de su banco de contenidos audiovisuales.

Estos dos criterios de programación contribuyen a la reflexión acerca de la concepción de los estados en relación con su rol en el conjunto del sistema de radiodifusión: por un lado, la historia de una política de comunicación proclive a la subordinación frente a los intereses 
privados y, por otro, de nuestra historia reciente y -a partir de la aprobación de la Ley 26522 de Servicios de Comunicación Audiovisual en 2009- un cambio de paradigma que se basó en una política de Estado que intentó con éxitos dispares construir un modelo cultural no mercantilizado que ofreciera nuevos propósitos narrativos, estéticos y políticos, luego de haber experimentado límites y contradicciones.

No obstante, la gestión del gobierno nacional que asumió a fines de 2015 en Argentina fijó inmediatamente criterios explícitos que promovieron la concentración de los grandes operadores privados en el marco de los procesos de integración y convergencia tecnológica a gran escala. Las modificaciones centrales que se operaron sobre la Ley 26522 fueron aplicadas para derogar la cuota de mercado de los grandes operadores y desarticular la composición multisectorial de la autoridad de aplicación, y ponerla bajo control absoluto del Poder Ejecutivo.

\section{Sobre la creación de los canales abiertos privados con fines de lucro}

La radiodifusión en la Argentina se implementó sobre la base del modelo comercial norteamericano en el contexto de las necesidades geopolíticas y de su influencia en la región, que culminó con la consolidación del sistema privado, comercial y de transmisión en cadenas. La emisión en red desde mediados de la década del treinta prefiguró un modo desigual y concentrado económico y geográfico en la producción y distribución de la programación de radio:

La televisión, con otra forma de nacimiento y posterior conformación societaria privada y extranjerizada, apenas andando a partir de principios de los 60 ya estableció su carácter privado en cadena, concentrado y desigual, bajo el control patrimonial y cultural de empresas norteamericanas (Pajoni, 2017).

\section{La radiodifusión gestionada por el Estado}

Van Cuilemburg y McQuail (2005) hicieron su aporte en la consideración de los dos modelos fundamentales en la constitución y consolidación de los sistemas de radiodifusión:

la norteamericana, que prioriza la entrega de licencias a actores privados, favoreciendo el 
desarrollo de un sistema mediático comercial, bajo control estatal; y la europea, con un histórico recorrido de gestión pública de medios estatales. Este modelo europeo incluía inicialmente características como la creación de corporaciones públicas para la gestión de los medios; el monopolio de los servicios de radio y televisión; el financiamiento asegurado a través de un canon; la obligación de la cobertura nacional total y de una programación balanceada. Este modelo europeo de corporación de SPR ha constituido el núcleo desde el cual surgieron los lineamientos básicos en la búsqueda de un modelo latinoamericano (Linares, 2014: 162).

Efectivamente, la doctrina de la Radiodifusión de Servicio Público (RPS) desde su conformación con el diseño de la BBC en Inglaterra, se convirtió en el punto de referencia frente a los modelos mercantiles y privados que nacieron a la luz de la experiencia norteamericana. La RSP se constituye a partir de objetivos político-culturales, subordinada a las políticas de Estado que son las encargadas de construir modelos de identidad nacional, cohesión social y estabilidad en el ordenamiento político.

Los diseños institucionales y las encrucijadas políticas latinoamericanas pusieron en evidencia las dificultades el cumplimiento de esos ideales en la gestión de los medios estatales latinoamericanos. Los alcances del modelo de servicio público contempla que el "derecho a la comunicación supone garantizar que el espacio público de la comunicación, donde actúan instituciones como los medios, está abierto realmente a todos las personas y grupos sociales" (Monje, 2006: 4-5). Cumplir con este propósito contribuye al mejoramiento de la democratización de los procesos de comunicación masiva porque opera como una "práctica que amplía las posibilidades de participación popular en la toma de decisiones sobre las políticas más globales" (Mastrini y Mestman, 1995: 2). Así, la radiodifusión de servicio público debe ser comprendida como un modo de organización específica -que no es la única y que es parte de una política pública de comunicación- de abordar y dar respuesta a ese interés público, entendido como valor a proteger y promover" (Linares, 2014: 160). Por su parte, si bien Gaetän Tremblay reconoce la dificultad de hallar una definición única de servicio público, aportó una definición que contiene el núcleo del modelo:

una actividad considerada de interés general por una colectividad y como tal reconocida por el Estado. Dicha actividad de interés general no puede abandonarse a la iniciativa privada y al puro juego de las leyes de mercado, y por tanto el Estado asume directamente su responsabilidad y su control, sometiéndola, si fuera necesario, a un régimen jurídico especial (1988). 
La expresión servicio público, para el caso de la radiodifusión, incluye en la experiencia latinoamericana a las emisoras y dispositivos de masas que operan bajo la lógica de servicios sin fines de lucro, "con algún tipo de propiedad pública, no necesariamente estatales -pero que generalmente son de propiedad estatal- y que intentan dar representación plural a la totalidad de los intereses de la sociedad" (Linares, 2014: 161). Focalizados en esta última dimensión, es útil recuperar que la noción incluye "a los medios gestionados directa o indirectamente por el Estado, los principios programáticos que los rigen y los legitiman en el conjunto del sistema de medios de un país (o región) y las normas que estructuran su organización y funcionamiento" (Valente, 2009: 25). Para el caso de Latinoamérica, los debates han expuestos la problemática de la gubernamentalización de las emisoras bajo gestión estatal, no solo en los gobiernos militares de facto, sino en los propios gobiernos democráticamente elegidos.

\title{
¿Un modelo latinoamericano?
}

\begin{abstract}
Cada país -comenzando por los europeos de vieja tradición en Servicio Público- maneja su propia definición de 'servicio público' en comunicaciones, así que una distinción razonablemente unívoca entre bien y servicio público o entre servicio estatal, gubernamental, oficial, cultural, alternativo, comunitario o de monopolio natural es por el momento difícil de establecer (Valente, 2005: 73).
\end{abstract}

Por otra parte, "el uso de la expresión public service broadcasting guarda sentidos distintos, siendo aplicado sólo a los medios públicos en algunos países o al conjunto de medios de concesionarios de canales de radio o de TV terrestres, como es el caso de Reino Unido" (2009: 25). En todo caso, es importante retener que la noción incluye "a los medios gestionados directa o indirectamente por el Estado, los principios programáticos que los rigen y los legitiman en el conjunto del sistema de medios de un país (o región) y las normas que estructuran su organización y funcionamiento" (Linares, 2013).

En una presentación realizada en Colombia Jesús Martín-Barbero presentó una declaración de principios donde señalaba que

la televisión pública debe ser el escenario del diálogo nacional intercultural (...), debe promover lo universal que no pasa por lo comercial (...), debe ganar el proceso y el flujo propio de las narrativas audiovisuales (...), debe hacer programas de gran impacto, que se conviertan en hechos sociales y ameriten repetición (...), debe ampliar las posibilidades 
simbólicas de representación, reconocimiento y visibilidad para la construcción de ciudadanía, sociedad civil y democracia (...), debe formar a los televidentes en los ámbitos de la lectura crítica de las imágenes y del control ciudadano sobre los mensajes audiovisuales que se exhiben en toda la televisión (...), debe programarse y producirse por convocatoria pública a través de procesos de asignación de espacios transparentes y participativos, en coherencia con las políticas culturales de comunicación y educación de cada país, y con base en los méritos de los realizadores y productores (2000).

Jesús Martín-Barbero (2001) defiende la idea de una TV pública que ofrezca "a todos los públicos un lugar de encuentro, así sea cambiante y precario" como en la reconstrucción de lo público y de allí a una apuesta por el proyecto público de la televisión "que, haciéndose cargo de las nuevas condiciones de producción y oferta, de las innovaciones tecnológicas y las reconfiguraciones de la audiencia", ofrezca los siguientes servicios: a. reconocimiento y expresión de la diversidad cultural; b. represente la pluralidad ideológico-política; c. promueva una información independiente, plural e incluyente de las diferentes situaciones regionales; $d$. debido a la fragmentación que introduce el mercado, se hace necesaria un televisión que se dirija al conjunto de ciudadanos de un país, que contrarreste en la medida de lo posible la balcanización de la sociedad nacional.

\section{Sobre la calidad y la diversidad cultural}

La gestación y consolidación de los dos modelos paradigmáticos de gestión, financiamiento y propuesta cultural en la radiodifusión nacen ya atravesados por una cuestión dilemática no resuelta: el sistema público promueve la calidad cultural y el sistema comercial entretiene en la persecución del lucro. El discurso de la calidad se presenta como un imperativo categórico para las televisiones estatales/públicas, mientras que los modelos comerciales legitiman su programación a partir del rendimiento económico de sus emisoras basados en una programación de entretenimiento propio de la comunicación de masas.

Esta diferencia para interpelar a los dos modelos genera debates acerca del modo de regulación en ambos casos. Richeri y Lasagni (2006) destacan dos posiciones: una de ellas promueve la necesidad de establecer "Reglas y obligaciones para todos que incluye a los canales privados a someterse a obligaciones y comportamientos de naturaleza social, política o cultural o pedagógica". La condición de empresas productoras de bienes culturales y simbólicos, operando a partir de una concesión otorgada por el Estado debe someterse a un 
conjunto de regulaciones porque "El entretenimiento no es el estado natural de la televisión, la televisión siempre aunque no se lo proponga es propagadora de ideas, de cultura y de valores, fatalmente"; mientras que otra perspectiva le otorga al sistema comercial la ventaja de juzgar su calidad en términos de eficiencia como empresa. ¿La televisión pública debe tener intereses sociales y de servicio, y la privada quedarse solo en la lógica del mercado? Richeri y Lasagni (2006) establecen una serie de obligaciones de las televisiones públicas: responsabilidad respecto de la calidad de su programación, respecto del público destinatario, responsabilidad política sobre un sistema informativo plural, entre otras.

Para Omar Rincón,

\begin{abstract}
La TV pública debe generar verdades plurales, crear relatos de identidad. Debe preguntarse sobre las experiencias de la gente, la vivencias culturales para la comprensión de la vida cotidiana; sirve para conectar a la gente alrededor de buenas ideas en la lucha por la dignidad, tácticas de visibilización de lo olvidado frente a lo comercial y constructora de conciencia pública (2001).
\end{abstract}

Sostiene que debe ofrecer expresiones que den cuenta de la lucha por las identidades " $y$ el reconocimiento de las comunidades en universos de mixtura cultural". El autor enumera un conjunto de aspiraciones para que la televisión pública cumpla con esos objetivos con propuestas de nuevos lenguajes, formatos, estilos, participación ciudadana y representación de la pluralidad ideológica.

No obstante, sopesar estos principios con la experiencia argentina pone en evidencia una innumerable cantidad de inconsistencias, porque

\begin{abstract}
la búsqueda de un modelo único y reconocidamente universal de la RSP se enfrenta a la dificultad de no contemplar -en su intento de caracterización ideal- las condiciones históricas de construcción de lo estatal y de la gestión de políticas públicas en contextos de desigualdad como se experimenta en los países periféricos, aunque el análisis no admite una justificación en los casos generalizados de la experiencia argentina donde se advierte el manejo arbitrario, gubernamentalizado y mercantilizado de la gestión (Pajoni, 2017).
\end{abstract}

\title{
Emisoras gestionadas por los estados provinciales
}

En la Argentina, el surgimiento y desarrollo de la radio en la década de 1920 y luego de la 
televisión no se adoptó ningún modelo de forma planificada de parte del Estado:

Si bien cada país presenta sus particularidades los inicios de la radiodifusión en casi todos los países de la región (con la excepción de Colombia y Chile) predominó un sistema netamente privado de carácter comercial. Paralelamente, se desarrollaron sistema de medios estatales que fueron puestos bajo la órbita de los gobiernos, quienes los usaron mayoritariamente para difundir sus políticas y discursos. Este uso de los medios estatales no contribuyó a fomentar el prestigio de los servicios públicos de radiodifusión en la ciudadanía latinoamericana (Mastrini, 2013: 61).

Retomando caracterizaciones precedentes, es necesario subrayar que

la creación de canales abiertos en las distintas provincias argentinas persiguió en cada momento objetivos que no atravesaron dilemas o debates acerca de la conveniencia de promover modelos públicos de radiodifusión o de ceder la explotación de las frecuencias a privados para consolidar un sistema comercial y competitivo entre privados (Pajoni, 2017).

Estas emisoras fueron creadas para alcanzar mayor cobertura territorial de la oferta cultural de la televisión central y privada, entonces "se promovieron indistintamente la creación de canales con prestadores privados o, cuando no los había, el propio estado provincial" (ibídem). Se verá más adelante que esta lógica se corrobora porque gran parte de los canales estatales reproducían los contenidos de los canales privados de la Ciudad de Buenos Aires.

El relevamiento de los contenidos de la programación de cada canal abierto de la Argentina en su área de cobertura permitirá establecer diferencias o semejanzas en la propuesta cultural de cada emisora bajo los parámetros conceptuales presentados hasta aquí, respecto de la función de cada emisora en su carácter de prestador privado o público.

\section{Programaciones, concentración y diversidad}

\section{Provincia de Córdoba}

\section{Canal 10, Universidad Nacional de Córdoba}

LV 80 Canal 10 inició sus transmisiones el 11 de mayo de 1962, convirtiéndose así en el segundo canal abierto que tuvo Córdoba y de las escasas experiencias argentinas de canales abiertos universitarios. 
A partir de 2007, el canal universitario retransmite contenidos educativos y periodísticos de las señales nacionales Canal 7 y Canal Encuentro. Desde 2009 amplió de 2,5 a 6 la cantidad de horas de programación dedicadas a los servicios informativos de producción propia.

Tiene noticieros y programas sobre actualidad informativa de la provincia de Córdoba. No reproduce noticieros de las canales de cabecera de la Ciudad de Buenos Aires. Los programas que no son de producción propia pertenecen a Canal 7 del Estado Nacional y a dos series extranjeras.

La representación de producciones de canales privados de la Ciudad de Buenos Aires es inexistente. No produce ficción propia.

\section{Licenciatarios privados con fines de lucro}

LV81. TV CANAL 12 de la empresa TELECOR perteneciente al Grupo Clarín y LV85. TV CANAL 8 operado por la empresa DICOR DIFUSION, perteneciente al Grupo Telefé/Viacom.

La programación de los canales privados verifica la existencia de dos procesos de distinto origen pero con el mismo resultado respecto de la concentración geográfica de la producción y la homogeneidad de los productos culturales generados en la ciudad de Buenos Aires. Por un lado, con el surgimiento de la televisión privada en la década del sesenta convertida en un sistema concentrado y de transmisión en cadena del centro a las distintas provincias más pobladas, y en la década del noventa con la conformación de multimedios y la concentración y transnacionalización del capital en el sistema de radiodifusión, en particular, con la existencia de los dos operadores dominantes del mercado: Grupo Clarín y CEI-Telefónica. Estos procesos explican el contenido de las programaciones privadas que replican programaciones ajenas y sin representación de la región de cobertura.

\section{Provincia de Tucumán}

LW83. TV Canal 10 Tucumán, operado por Televisora de Tucumán S.A.P.E.M.

Canal 10 comenzó sus transmisiones el 9 de julio de 1966. En 1977 se conformó la sociedad anónima Televisora de Tucumán S.A.P.E.M. (Sociedad Anónima con Participación Estatal Mayoritaria). Pertenece a la Universidad Nacional de Tucumán en un $70 \%$ y al gobierno provincial en el 30\%. Desde finales de los noventa hasta 2003 transmitía con exclusividad la programación de Canal 13 de la ciudad de Buenos Aires, y actualmente también incluye programación del canal educativo Pakapaka y del canal Encuentro (ambos canales pertenecientes al Estado Nacional). 
Licenciatarios privados con fines de lucro

LRK458, TV Canal 8. Televisora Tucumana S.A. (Grupo Telefé/Viacom)

En el caso del canal estatal/universitario, los noticieros de actualidad informativa pertenecen a la programación de Canal 13 de la Ciudad de Buenos Aires, con la excepción de los días miércoles con una programación local: "Panorama tucumano", "Resto del mundo" y "De noche con Miguel Martin". A partir de las 13 horas y hasta el cierre de la transmisión reproduce la programación del mismo canal central. Carece de producción de ficción propia.

Por otra parte, la emisora privada produce informativos locales al contrario de la emisora sin fines de lucro, y reproduce la programación de su canal de cabecera: Telefé de la Ciudad de Buenos Aires.

\section{Provincia de Tierra del Fuego}

La provincia no tiene prestadores privados de servicios de televisión abierta. Por un lado, esta ausencia se relaciona con la historia de la conformación de los canales estatales y sus diversos contextos de surgimiento, y a razones de índole topográfica, demográfica y económica que desalentaron la existencia de operadores privados. No obstante, en gran parte de la historia de los dos canales estatales de la provincia se reprodujo la programación de canales privados de la Ciudad de Buenos Aires.

Canal 13 de Río Grande (Provincia de Tierra del Fuego)

Comenzó a transmitir el 24 de octubre de 1967, con una programación provista en su mayor parte por la productora Proartel (asociada a Canal 13 de la Ciudad de Buenos Aires) y se complementaba con documentales y posteriormente con programas locales. A partir de 1991 transmite la programación de Canal 11/Telefé de la Ciudad de Buenos Aires.

Canal 11 Televisión Pública Fueguina (Provincia de Tierra del Fuego)

Comenzó su transmisión oficial el 23 de octubre de 1967. A partir de 2012 dejó de retransmitirse la programación del Canal 13 de la Ciudad de Buenos Aires y comenzó a emitir contenidos provistos por los bancos de producción audiovisual del gobierno nacional.

\section{Provincia de Santa Cruz}

Canal del estado provincial, LU85 TV Canal 9. Rio Gallegos, Santa Cruz.

La provincia no tiene prestador privado de televisión abierta. En 1968 se autorizó a la Provincia 
de Santa Cruz a prestar un servicio de radiodifusión de televisión abierta, a través de la emisora LU 85 TV Canal 9 de Río Gallegos.

El 28 de septiembre de 1989, la legislatura provincial aprobó la Ley 2136 en la que se le otorgó un estatuto jurídico y funcional. De este modo, se dispuso la creación del Canal Provincial de Televisión como "un Ente con Autarquía funcional y financiera", conducido por "un Directorio integrado por un (1) Presidente y tres (3) Vocales", con predominio de la representación del poder ejecutivo provincial.

La programación tiene un $50 \%$ de producción local, incluyendo informativos y programas de variedades, no transmite contenidos de los canales de la Ciudad de Buenos Aires, reserva un espacio para la producción de contenidos de la universidad tecnológica nacional de la región, y emite películas extranjeras. El resto de la programación pertenece a Canal 7 del Estado nacional y las señales de contenidos públicas, como Pakapaka y Encuentro.

\section{Provincia de La Pampa}

Canal 3 del Estado provincial y TV CPE (Canal de la Cooperativa Popular de Electricidad). El canal que opera bajo la modalidad de prestador sin fines de lucro ofrece una programación cuyos contenidos representan centralmente problemáticas locales. Sin ficción propia, programa la ficción de la oferta cultural de Canal 7 del Estado nacional.

El canal estatal refleja la mayor representación de programación propia de los estados provinciales de la Argentina, con más contenidos locales y regionales (no de canales de cabecera) y la mayor variedad de contenidos diferenciados en cada uno de los días de la semana, con representación de contenidos locales, regionales y centrales. No cuenta con noticieros de los canales de la capital del país.

La provincia no cuenta con operadores privados de televisión abierta y el conjunto de la programación del canal estatal es local con escasas excepciones, como la señal para niños/as del estado nacional Pakapaka y Cocineros Argentinos y Cromo, también del Canal 7. La producción de informativos es propia, al igual que el resto de la programación.

El caso de La Pampa es un caso excepcional donde la programación de los canales de la capital argentina no tiene presencia en la oferta de contenidos de los canales abiertos.

\section{Provincia de Misiones}

Canal 12 del estado de la Provincia de Misiones

Al igual que en las otras experiencias, el canal retransmitía programas de Canal 13 de la Ciudad de Buenos Aires. A partir de 2010, la programación adquirió otra propuesta 
radicalmente diferente, integrada por contenidos de Canal 7 y señales de contenidos también producidos por el Estado nacional. Asimismo, la producción local la componen los programas informativos y otros magazines diarios representativos del área de cobertura.

Canal privado con fines de lucro

Canal 2 de Posadas, Misiones, que a partir del 1 de diciembre de 2011 transmite en directo la señal de Telefé. Reproduce la programación de Telefé de la Ciudad de Buenos Aires con excepción de tres ediciones de los noticieros locales.

\section{Provincia de San Luis}

TV Canal 13 del estado de la provincia de San Luis

La primera transmisión se realizó el 24 de diciembre de 1972. Existía hasta ese momento el Canal 2 de circuito cerrado que luego se convirtió en la base de la experiencia y la infraestructura para poner al aire Canal 13 , realizando el itinerario ya caracterizado que refleja el propósito del gobierno militar de la época de neutralizar el avance de los circuitos cerrados de televisión paga e instalar canales abiertos que garanticen mayor control sobre la gestión y los contenidos.

La propuesta de programación no constituye cadena de emisión con canales abiertos de la ciudad de Buenos Aires, ni siquiera existen contenidos generados en la capital del país. Se registra producción informativa propia, magazines locales y programas de cadenas internacionales.

\section{Provincia de Chaco}

Canal 28 Chaco TV, canal del estado de la provincia de Chaco.

Nació en 2010 en el marco de la vigencia de la Ley de Servicios de Comunicación Audiovisual 26522, con el otorgamiento de frecuencias para universidades, municipios y provincias.

Canal con fines de lucro

Canal 9

El Canal 9 de Resistencia inició sus transmisiones el 17 de agosto de 1966. A partir de octubre de 2007, está bajo control del Grupo Clarín y emite la programación del Canal 13 de Buenos Aires (perteneciente al mismo conglomerado de medios de comunicación). 


\section{Provincia de La Rioja}

Canal 9 del Estado provincial

Su programación retransmite los contenidos del Canal 11 de la Ciudad de Buenos Aires. A partir de 2009, comenzó a incluir programación de las señales de contenidos públicas, en el marco del Sistema Federal de Medios Públicos organizado por el gobierno nacional de entonces.

\section{Provincia de Formosa}

Canal 11 Lapacho TV del Estado provincial

Con escasa programación propia, los programas vespertinos y nocturnos -incluyendo el noticiero central- del prime time pertenecen a la oferta de Canal 11 Telefé de la Ciudad de Buenos Aires.

\section{Provincia de Chubut}

Canal 7 Rawson del Estado provincial

Inició sus transmisiones el 15 de septiembre de 1975. La programación retransmitía el conjunto de los contenidos del Canal 13 de la Ciudad de Buenos Aires, a partir de 2009 se incorporó al sistema público con la programación de la TV Pública, Encuentro y Pakapaka, además de programas de producción local.

\section{Provincia de Río Negro}

Canal 10 del Estado provincial

El 14 de diciembre de 1998, R.T.R.N. se asoció con Canal 13 de la Ciudad de Buenos Aires para reproducir su programación, contrato vigente entre el 10 de marzo de 1999 y el 9 de marzo de 2009. El 10 de marzo de 2009, el gobierno provincial recuperó la administración de la empresa y se inscribió dentro del sistema federal de medios públicos. A partir de 2014 comenzó a denominarse Televisión Rionegrina.

\section{Los canales creados en el marco de la Ley de Servicios de Comunicación Audiovisual}

La creación de los canales abiertos se realizó en el marco regulatorio regido por la Ley de Servicios de Comunicación Audiovisual que autoriza a los estados provinciales a contar con un canal de aire, una emisora de Amplitud Modulada y una de Frecuencia Modulada. 
Acequia TV, provincia de Mendoza

La primera transmisión fue el 2 de marzo 2013, pero comenzó a emitir desde el Canal 29 de la Televisión Digital Abierta (TDA) el 26 de marzo de 2014. Es parte de la Subsecretaría de Comunicación Pública del Gobierno de Mendoza y se rige a través de un decreto firmado por el gobernador. El gobierno provincial tiene la facultad de designar al director de la emisora. Tiene producción local y orientada a la formación educativa, con programación en horario central de documentales, referencias históricas y científicas, entrevistas, informativos propios y contenidos de señales producidas por el Estado Nacional.

\section{Catamarca Radio y Televisión Sociedad del Estado}

La empresa Catamarca Radio y Televisión Sociedad del Estado fue inaugurada el 24 de abril de 2014 en el marco de la vigencia de la Ley de Servicios de Comunicación Audiovisual. Programa dos noticieros locales diarios, magazines e programas de actualidad propios y se inscribe en la programación de contenidos producidos por el gobierno nacional.

\section{Resultados}

A partir del relevamiento de la programación diaria de cada emisora, se advierte una constante que aparece como característica central del diseño de la propuesta de contenidos: la ausencia o presencia de canales privados, siempre vinculados patrimonialmente o asociados a la programación central de los canales de cabecera de la Ciudad de Buenos aires determina el contenido de los canales estatales/universitarios.

Esto revela que la definición de la programación, en su mayoría, obedece a aspectos económicos y políticos: por un lado, a la provisión de productos mainstream que garantizan la demanda de las audiencias, y a la vez se ajusta a modelos de negocios que se sustentan con la reproducción de programación ajena (alquiler de antena) sin inversión, pues son los bienes producidos por los canales de cabecera de la CABA.

Se advierte que la programación del canal estatal cambió sustancialmente en pocos años su propuesta de contenidos, más sujeta a los vaivenes de la conducción política de la nación que inspirada en una propuesta cultural de largo plazo. La dificultad de financiamiento y sustentabilidad del canal lo somete a la dinámica de las disputas políticas y la dirección económica provincial y nacional, subordinando cualquier otro aspecto cultural a esas urgencias. Del análisis riguroso de las propuestas de contenido de cada canal abierto de televisión analizado en este trabajo, surgen cinco tipologías de casos. Estos modelos de programación 
no son simples catálogos elaborados para ordenar la presentación de este estudio, sino que son diseños que aparecen en la superficie de cada una de estas experiencias analizadas de manera elocuente y que se definen a partir de la presencia de licenciatarios privados en el área de cobertura donde opera cada canal de los estados provinciales.

\section{Caracterización por sistema de medios existente}

\section{Caso 1. Programación del canal estatal, definida por la presencia de dos prestadores privados}

La emisora universitaria de la provincia de Córdoba tiene como competidores en el mismo área a los canales privados de la CABA: Canal 12 perteneciente al Grupo Clarín; y Canal 8, perteneciente al grupo Viacom, licenciatario de Telefé, Canal 11 de la CABA.

Este diseño exige a las emisoras sin fines de lucro estatales o universitarias -que conviven en esos mercados- propuestas de programación alternativas que no se repiten en las zonas en las que no operan privados.

\section{Caso 2. Programación del canal estatal, definida por la presencia de un solo prestador privado}

El caso del canal abierto de la universidad de la provincia de Tucumán transmitía con exclusividad la programación de Canal 13 de la CABA, y a partir de 2003 incluye también programación del canal educativo Pakapaka y del canal Encuentro (ambas señales pertenecientes al Estado Nacional). A partir de las 13 horas y hasta el cierre de la transmisión reproduce la programación del mismo canal central, y carece de producción de ficción propia. La existencia en la misma área de cobertura del canal privado, cuya titularidad le pertenece a Telefé (Canal 8), explica el predominio de los contenidos de Canal 13 de la CABA sobre la programación del canal estatal/universitario de Tucumán.

En este caso, se da la particularidad de que la emisora privada produce informativos locales, mientras que por el contrario de lo que indicaría la lógica no mercantil, la emisora sin fines de lucro reproduce los noticieros del canal de la CABA (con la excepción de los días miércoles con una programación local: "Panorama tucumano", "Resto del mundo" y "De noche con Miguel Martin") a espaldas de la representación de área primaria de cobertura.

Se manifiesta de este modo, el inesperado resultado de que los canales locales sin fines de lucro en mucho casos no representan ni programan en función de intereses regionales, sino 
que sus licenciatarios gestionan contenidos sin principios reguladores propio de su función cultural, sino que prima el criterio mercantil; que por otra parte es propio del proceso histórico y político que les dio nacimiento.

El caso de la provincia de Misiones se inscribe en esta lógica del caso 2, con la coexistencia del Canal 12 del Estado de la Provincia de Misiones y Canal 2 de Posadas, que opera una licencia con fines de lucro. El canal estatal hasta 2010 retransmitía el conjunto de la programación de Canal 13 de la Ciudad de Buenos Aires. Mientras que Canal 2, especialmente a partir del 1 de diciembre de 2011 llega a un acuerdo de programación exclusiva para reproducir la programación de Telefé. Pero a partir de 2010, la programación adquirió otra propuesta radicalmente diferente, integrada por contenidos de Canal 7 y otros también producidos por Estado nacional. Asimismo, la producción local la componen los programas informativos y otros magazines diarios representativos del área de cobertura.

\section{Caso 3. Programación con dos canales estatales sin competencia privada}

El caso de la provincia de Tierra del Fuego explica la ausencia de prestadores privados por la escasa rentabilidad de la región, y la presencia de canales estatales por la necesidad del Estado Nacional de generar cobertura en zonas fronterizas. En el particular caso fueguino, con dos canales estatales gestionados por el gobierno provincial (Canal 13 de Río Grande y Canal 11 Televisión Pública Fueguina) la programación se sostuvo con acuerdos de transmisión de la programación en forma exclusiva de cada canal de la CABA, Canal 13 y Telefé respectivamente.

En 2012 dejó de emitirse la programación del Canal 13 de Buenos Aires y comenzó a emitir contenidos provistos por los bancos de producción audiovisual promovidos por el gobierno nacional en el contexto de la aplicación de la Ley de Servicios de Comunicación Audiovisual.

\section{Caso 4. Programación propia sin prestadores privados locales ni acuerdos de programación con los canales privados de la CABA}

La provincia de La Pampa ofrece un marco singular respecto de las características generales que describen la dinámica de la televisión provincial en la Argentina. Operan tres canales abiertos de televisión sin ninguna relación patrimonial o de programación con los canales privados de la CABA. Así el mapa de la televisión pampeana incluye:

-Canal 3 del Estado provincial. 


\section{-Canal de la Cooperativa Popular de Electricidad TV CPE}

-Canal 6: de origen comunitario y en la actualidad transita un complejo proceso de delegación de su explotación en manos privadas. Se trata de un contrato de alquiler, cuyo locatario es representante de la empresa del Grupo Clarín, quien a través de las empresas Multicanal y Teledigital monopoliza el mercado del cable local.

El canal TV CPE opera bajo la modalidad de prestador sin fines de lucro y ofrece una programación cuyos contenidos representan centralmente problemáticas locales. Sin ficción propia, programa la ficción de la oferta cultural de Canal 7 del Estado nacional. Por otro lado, el Canal 3 estatal refleja la mayor representación de programación propia de los estados provinciales de la Argentina, con más contenidos locales y regionales y la mayor variedad de contenidos diferenciados en cada uno de los días de la semana, además de no programar noticieros de los canales privados de la CABA.

El caso de la provincia de San Luis también se inscribe en esta casuística. El Canal 13 del estado provincial ofrece contenidos en su programación que no constituyen cadena de emisión con canales abiertos de la capital del país, sino contenidos de origen local al igual que informativos propios con material de ficción de cadenas internacionales.

Responde a la misma lógica de programación, pues hay una alta representación de contenidos locales, al igual que los contenidos informativos. Por otro lado, está inscripto en el sistema federal de medios públicos con programación generada por el estado nacional.

\section{Caso 5. Programación del canal estatal, definida por la transmisión en red de contenidos de canal privado de la CABA.}

En la provincia de Chubut con Canal 7 operado por el estado, la programación consistía en la retransmisión del conjunto de los contenidos del Canal 13 de la CABA (a partir de 2009 se incorporó al sistema público con la programación de la TV Pública, Encuentro y Pakapaka, además de programas de producción local).

Es el mismo caso de Canal 9 operado por el gobierno la provincia de La Rioja. Su programación retransmite los contenidos del Canal 11/Telefé de la CABA. A partir de 2009, comenzó a incluir programación de las señales de contenidos públicas.

El Canal 10 operado por la provincia de Río Negro se asoció con Canal 13 de la Ciudad de Buenos Aires para reproducir su programación, contrato vigente entre el 10 de marzo de 1999 y el 9 de marzo de 2009. El 10 de marzo de 2009, el gobierno provincial recuperó la administración de la empresa y se inscribió dentro del Consejo Federal de la Televisión Pública 
del gobierno nacional. A partir de 2014 comenzó a denominarse Televisión Rionegrina.

El caso de Canal 11 Lapacho TV de la provincia de Formosa se inscribe en esta lógica de acuerdos de programación con canales de cabecera. Fue inaugurado el 8 de abril de 1978, bajo control del Estado Nacional y mantiene por fuera de los horarios centrales de programación, contenidos de programación propia. Los programas vespertinos y nocturnos incluyendo el noticiero central- del prime time pertenecen a la oferta de Canal 11 de la CABA.

\section{Canales creados en el marco de la vigencia de la Ley de Servicios de Comunicación} Audiovisual

La creación de estos canales abiertos se realizó en el marco regulatorio establecido por la Ley 26522 de Servicios de Comunicación Audiovisual donde se autorizó a los estados provinciales a contar con un canal de aire, una emisora de Amplitud Modulada y una de Frecuencia Modulada. Su programación está definida por la producción pública de contenidos audiovisuales fomentadas por el gobierno nacional hasta 2015. Los organismos destinados a la producción de contenidos audiovisuales de carácter estatal experimentan hoy un claro retroceso de producción y presupuestario.

\section{Acequia TV Provincia de Mendoza}

La primera transmisión fue el 2 de marzo 2013, pero comenzó a emitir desde el Canal 29 de la Televisión Digital Abierta (TDA) el 26 de marzo de 2014. Es parte de la Subsecretaría de Comunicación Pública del Gobierno de Mendoza y se rige a través de un decreto firmado por el gobernador. El gobierno provincial tiene la facultad de designar al director de la emisora.

\section{Catamarca Radio y Televisión Sociedad del Estado}

La empresa Catamarca Radio y Televisión Sociedad del Estado fue inaugurada el 24 de abril de 2014 en el marco de la vigencia de la Ley de Servicios de Comunicación Audiovisual.

\section{Canal 28 Chaco TV}

Nació en 2010 en el marco de la vigencia de la Ley de Servicios de Comunicación Audiovisual 26522, con el otorgamiento de frecuencias para universidades, municipios y provincias. La resolución 155/2011 del AFSCA asignó, para el funcionamiento del servicio, el Canal 28 en la ciudad de Resistencia.

Existe en esta provincia el Canal 9 de Resistencia en la provincia de Chaco a partir de octubre 
de 2007 está bajo control del Grupo Clarín y emite la programación del Canal 13 de la CABA.

\section{Algunas lecturas posibles}

Los resultados presentados demuestran fehacientemente que en la historia de la radiodifusión argentina el carácter estatal o la explotación privada de la operación de cada canal de televisión no determina el modelo cultural de su propuesta de contenidos. Esta conclusión es resultado también de la forma de constitución de estas emisoras, en cuya génesis se manifiestan objetivos de índole político y de cobertura territorial para abastecer a la población de los consumos culturales masivos producidos en la cápita del país.

No es sorprendente entonces, con la vista puesta en el origen de estas emisoras, la ausencia de principios fundacionales de carácter educativo y cultural que constituyen los ideales de las formas estatales en la gestión de medios de comunicación. Así, los acuerdos de programación entre las emisoras estatales y los canales privados de televisión abierta de la Ciudad de Buenos Aires se constituyen en una característica fundamental del modelo.

Se observan en los distintos casos presentados, que el canal estatal ofrece la programación del canal privado de la ciudad de Buenos Aires siempre y cuando en la misma área de cobertura opere su competencia más importante. Es decir que los dos canales abiertos más relevantes de la capital del país se disputan el mercado de otros territorios, no sólo a través del control patrimonial de canales provinciales, sino a través de las mismas emisoras estatales, subrayándose un despropósito político y cultural que va a contramano del rol de los estados frente a la producción de bienes culturales masivos y mercantilizados, como se mencionó en los principios que guían el rol de la radiodifusión estatal/pública.

No obstante, como se registró en el relevamiento, existen escasas experiencias en zonas sin participación de licenciatarios privados que la propuesta cultural del canal de gestión estatal no obedece a la lógica mencionada, sino a propuestas culturales más representativas de la idiosincrasia regional; en ningún caso se advierte una gestión independiente del poder gubernamental, habida cuenta de la existencia de dependencia administrativa directa del director del canal respecto del poder ejecutivo local.

Asimismo, se advierte que la programación de las televisiones estatales cambió sustancialmente en pocos años, más sujeta a los vaivenes de la conducción política de la nación que inspirada en una propuesta cultural de largo plazo. La dificultad de financiamiento y sustentabilidad del canal lo somete a la dinámica de las disputas políticas y la dirección 
económica provincial y nacional, subordinando cualquier otro aspecto cultural a esas urgencias. Es ineludible mencionar el cambio de programación que experimentaron el conjunto de estos canales abiertos a partir de la aprobación de la ley de Servicios de Comunicación Audiovisual. Para una clara interpretación de los cambios estructurales que se están produciendo en el terreno de la producción y la distribución de los servicios de comunicación audiovisual es que, en todos los casos, los canales estatales que retransmitían en red los contenidos de los canales privados de la CABA, a partir de 2009 modificaron su programación y se inscribieron a la pauta de contenidos organizada por el sistema Federal de Medios y Contenidos Públicos creado en el marco de la aplicación de la Ley de Servicios de Comunicación Audiovisual. Es así como se advierte -ya en forma de política de Estado consolidada- un cambio a partir de 2010, con una programación compuesta por la TV Pública del Estado Nacional y sus señales educativas Encuentro, Depor TV y Pakapaka, y los contenidos aportados por el Banco Audiovisual de Contenidos Audiovisuales Argentino (BACUA) y el Árbol de Contenidos Audiovisuales Argentino (ACUA).

No obstante, se produce en esta coyuntura un nuevo cambio de paradigma con el advenimiento del nuevo gobierno nacional a partir de diciembre de 2015, donde las nuevas políticas de estado en torno a la inversión pública en la producción de contenidos se ven marcadamente reducidas, a partir de una concepción que presenta sustanciales cambios en torno al rol del Estado y sus modos de intervención.

A partir de las modificaciones aplicadas a la Ley 26522, se creó el Sistema Federal de Medios y Contenidos Públicos (SFMCP) dependiente del Poder Ejecutivo nacional. Además, el decreto 1222 de 2016 creó Contenidos Públicos Sociedad del Estado, dependiente del SFMCP, a donde se transfiere Encuentro, Pakapaka, Depor TV, Polo de Producción Audiovisual y el Banco Audiovisual de Contenidos Universales Argentinos (BACUA).

Los contenidos de estas señales y usinas de producción de contenidos audiovisuales han sufrido una reducción drástica de sus presupuestos y de sus empleados, lo que implicará en un corto plazo un cambio en la programación de los canales de los estados provinciales que aún no se ha expresado formalmente, y que promoverá nuevas dinámicas para las necesidades de programación local.

La historia de la radiodifusión estatal argentina no ha superado la lógica mercantil, ni se ha constituido como un agente que equilibre la oferta de las narrativas, la representación de la producción cultural de cada región, ni ha perseguido principios de independencia de los poderes de turno. El relevamiento de la programación expone grandes déficits en la radiodifusión estatal, y una clara subordinación de los principios de la diversidad cultural a los 
de la sustentabilidad económica y política.

Los cambios operados a partir de 2009 no están garantizados en su continuidad cuando los principios mercantiles, y la inversión pública presenta hoy grandes restricciones que regirán en el futuro inmediato las políticas de Estado.

\section{Bibliografía}

Arroyo, L.; Becerra, M.; García Castillejo, A. y Santa María, O. (2012). Cajas mágicas. El renacimiento de la televisión pública en América Latina. Madrid: Editorial Tecnos.

De Charras, D.; Lozano, L. y Rossi, D. (2013). Ciudadanía(s) y derecho(s) a la comunicación. En Mastrini, G.; Bizberge, A. y de Charras D. (eds.). Las políticas de comunicación en el siglo XXI (pp. 25-52). Buenos Aires: La Crujía.

Getino, O. (1995). Las Industrias Culturales en Argentina: dimensión económica y políticas públicas. Período 1981-1992. Buenos Aires: Colihue.

Mata, M. C. (octubre de 2012). Panel de Apertura. X Encuentro Nacional de Estudiantes de Comunicación. ENEC-ELAC, Córdoba.

Mastrini, G. (2013). Medios públicos y derecho a la comunicación. En Mastrini, G.; Bizberge, A. y De Charras, D. (Eds.). Las políticas de comunicación en el siglo XXI (pp. 53-74). Buenos Aires: La Crujía.

Mastrini, G. y Mestman, M. (1995). ¿Desregulación o rerregulación? De la derrota de las políticas a las políticas de la derrota. I Jornadas de Jóvenes Investigadores en Comunicación de Buenos Aires, Buenos Aires.

Mastrini, G.; Becerra, M. y Marino, S. (2010). El proceso de regulación democrática de la comunicación en Argentina. Oficios Terrestres, 25, 11-24, Facultad de Periodismo y Comunicación Social, Universidad Nacional de La Plata.

Mastrini, G.; Becerra, M. y Marino, S. (2012). Mapping Digital Media: Argentina. Nueva York: Open Society Foundations.

Monje, D. (2006). Apuntes para el estudio de Políticas de Radio y TV frente a procesos de integración regional. Caso MERCOSUR. UNIrevista, 3, 1-10, Río de Janeiro.

Organización de Estados Americanos (2010). Estándares de libertad de expresión para una radiodifusión libre e incluyente. Relatoría Especial para la Libertad de Expresión. Comisión Interamericana de Derechos Humanos. Recuperado de http://www.cidh.org/relatoriaTremblay, G. (1988). La noción de servicio público. Revista 
Telos, 14, pp.57-63, Madrid.

Pajoni, H. (2017). Dependientes y mercantilizados: génesis, evolución y actualidad de los canales de televisión de los estados provinciales. Revista Question, /(55), julioseptiembre, pp.

81-99.

Recuperado

de http://perio.unlp.edu.ar/ojs/index.php/question/article/view/4238/3553

Pasquali, A. (1991). ¿Qué es una radiodifusión de servicio público? En El Orden Reina. Monte Ávila: Caracas.

Pasquali, A. (1995). Reinventar los servicios públicos. Revista Nueva Sociedad, 140, pp. 70-89.

Richeri, G. y Lasagni M. C. (2006). La calidad de la red. En Televisión y calidad, el debate internacional (cap. 3). Buenos Aires: La Crujía ediciones.

Rincón, O. (2001). Televisión pública: para saber quiénes somos, como hemos venido siendo y que queremos ser. En Rincón, O. (comp.). Televisión pública, del consumidor al ciudadano. Bogotá: Ed. Convenio Andrés Bello.

Romano, S. (2002). Política, Universidad y Medios: aportes para una historia de las condiciones de producción de noticias de Canal 10 de Córdoba (1958/1980). En Política, Universidad y Medios: contribución al estudio de las condiciones de producción de noticias de Canal 10 de Córdoba en los 60 y 70. Córdoba, Argentina: Ferreyra editor.

UNESCO (2005). Convención sobre la protección y promoción de la Diversidad de las Expresiones Culturales París. UNESCO. Recuperado de http://unesdoc.unesco.org/images/0014/001429/142919s.pdf

Valente, J. (2009). Concepçoes e abordagens conceituais sobre sistema público de comunicação Social. En Intervozes-Coletivo Brasil de Comunicação Social (Ed.). Sistemas públicos de comunicação no mundo. Experiencias de doze paises e o caso brasileiro (pp. 25-46). Intervozes/Paulus: San Pablo. 